modèles $\cdot \cdots \cdot \ldots+$ !

ling uistiques

\section{Modèles linguistiques}

$53 \mid 2006$

La préposition en français (I). Philologie et linguistique diachronique (domaine anglais)

\title{
8. De la difficulté à distinguer entre groupes prépositionnels régis et non régis
}

\section{Belinda Lavieu}

\section{OpenEdition}

\section{Journals}

Édition électronique

URL : https://journals.openedition.org/ml/527

DOI : $10.4000 / \mathrm{ml} .527$

ISSN : 2274-0511

Éditeur

Association Modèles linguistiques

Édition imprimée

Date de publication : 1 janvier 2006

Pagination : 131-144

Référence électronique

Belinda Lavieu, «8. De la difficulté à distinguer entre groupes prépositionnels régis et non régis »,

Modèles linguistiques [En ligne], 53 | 2006, mis en ligne le 01 février 2015, consulté le 01 juillet 2021.

URL : http://journals.openedition.org/ml/527 ; DOI : https://doi.org/10.4000/ml.527

Ce document a été généré automatiquement le 1 juillet 2021.

(C) Modèles Linguistiques 


\title{
8. De la difficulté à distinguer entre groupes prépositionnels régis et non régis ${ }^{1}$
}

\author{
Belinda Lavieu
}

\section{Introduction et problématique}

1 La grammaire dite "traditionnelle » - qui correspond au patrimoine scolaire depuis le $\mathrm{XIX}^{\mathrm{ème}}$ siècle et a défini la doxa grammaticale jusqu'aux années soixante - distinguait trois grands types de constituants dans la phrase : le sujet, le complément, l'attribut, le complément pouvant être d'objet (direct ou indirect) ou circonstanciel. La distinction entre les deux types de complément s'opérait par le critère de la question (à quoi s'ajoutait une définition sémantique) : qui est-ce que, qu'est-ce que, à qui, de quoi, etc. dans le premier cas, où, quand, comment, combien, pourquoi, essentiellement, dans le second.

2 La linguistique structurale, et à sa suite générativiste, privilégiant les propriétés syntaxiques, a progressivement imposé une nouvelle répartition des compléments en distinguant compléments de verbe, dits " essentiels ", et compléments de phrase, dits « inessentiels» (ces derniers souvent assimilés, à tort, aux anciens compléments circonstanciels tandis que les premiers le sont aux anciens compléments d'objets, direct et indirect) :

Parmi les compléments du verbe, on distingue le complément d'objet (direct et indirect), ces deux sortes de compléments ont valeur de complément essentiel, et le complément circonstanciel, non-essentiel, qui s'oppose aux compléments essentiels dont il se distingue par sa mobilité (Wagner \& Pinchon, 1962 : 24).

Les premiers types de compléments sont caractérisés par le fait qu'ils sont éventuellement supprimables, en tous cas non déplaçables, mais pronominalisables en le / la / les (Il les mesure), en (J'en reviens pour Je reviens de Paris) ou encore y (J'y vais pour Je vais à la pêche), tandis que ceux du second type sont définis comme étant 
supprimables et déplaçables, mais non pronominalisables. Cependant, la caractérisation n'est toujours pas parfaite puisque :

a. certains compléments de verbe sont supprimables, ce qui rend l'appellation "essentiel » quelque peu paradoxale et supprime ou relativise la pertinence de la propriété : Elle fume (des cigarettes). On sait en effet que les verbes normalement suivis d'un complément régi sont susceptibles de connaître l'emploi dit «absolu ». De plus, le résultat du test se décide sur la base d'une intuition sémantique (celle que la suppression du complément change ou non le sens du verbe), or celle-ci n'est pas forcément claire (le critère n'est en tout cas pas reproductible); par exemple, dans Elle lave son linge et Elle lave son linge à la machine, certes il s'agit dans les deux cas de «laver son linge » mais on n'a pas affaire à la même « action » : ainsi va-t-on dire ou non que à la machine est supprimable?

b. la pronominalisation ne concerne pas systématiquement les compléments de verbe : dans L'ennemi marche sur la ville, le syntagme prépositionnel sur la ville ${ }^{2}$ est un complément puisqu'il n'est pas supprimable sans changement de sens du verbe: marcher sur a ici l'interprétation "aller au combat », ce qui n'est pas le cas de L'ennemi marche; mais le SP n'est pas pronominalisable:??L'ennemi y marche; en revanche la pronominalisation est possible pour les compléments spatiaux de phrase (A Paris, il pleut > Il y pleut).

De fait, aujourd'hui les chercheurs remettent en cause l'opposition entre complément de verbe et complément de phrase, cette dichotomie faisant désormais place à une tripartition : on distingue maintenant, sous les anciens "compléments de verbe », en fait deux types de constituants, l'un qu'on appelle «adjoint» ou «ajout» ou « modifieur » ou encore « complément de SV » - du type sur le quai dans Elle a embrassé sa mère sur le quai ${ }^{3}$ - par opposition au " complément de verbe » tel que sur la joue dans Elle a embrassé sa mère sur la joue, qui, lui, est régi par le verbe - c'est-à-dire entièrement programmé par lui (sur la joue est un argument du verbe). Ce qui a la fonction de " compléter » peut apparaître à trois niveaux dans l'arbre syntaxique : rattaché à la phrase, au syntagme verbal ou au verbe. La terminologie est, dans ce domaine, prolifique et il est nécessaire de clarifier les choses en adoptant des dénominations univoques, d'autant que les termes ajout, complément, argument et modifieur ont pu être utilisés dans des acceptions différentes. Convenons pour notre part que nous parlerons de :

\begin{tabular}{|l|l|}
\hline $\begin{array}{l}\text { TERMINOLOGIE } \\
\text { SEMANTIQUE }\end{array}$ & $\begin{array}{l}\text { TERMINOLOGIE } \\
\text { SYNTAXIQUE }\end{array}$ \\
\hline $\begin{array}{l}\text { Complément : rôle sémantique de l'argument du verbe (si } \\
\text { cet argument n'est pas le sujet) }\end{array}$ & $\begin{array}{l}\text { Argument : constituant régi, i.e. faisant } \\
\text { partie de la construction définitoire } \\
\text { d'un lexème }\end{array}$ \\
\hline $\begin{array}{l}\text { Modifieur: fonction sémantique assurée par l'ajout } \\
\text { Modalisateur : fonction sémantique assurée par l'incident } \\
\text { - ce qu'on appelle également «adverbe d'énonciation » } \\
\text { (Le Goffic 1993) }\end{array}$ & $\begin{array}{l}\text { Ajout (de GV, de P) : constituant non régi } \\
\text { par opposition à l'argument }\end{array}$ \\
\hline
\end{tabular}

5 La différence est d'ordre syntaxique; soit le syntagme est intégré dans la phrase : il est intraphrastique ; soit il n'est pas intégré à la phrase (ce n'est pas un constituant), il porte 
sur l'intégralité de la phrase, qu'il modalise de l'extérieur: il est extraphrastique (Guimier 1996 utilise les termes (sémantiques) intraprédicatif et extraprédicatif, respectivement). L'opposition entre intraphrastique et extraphrastique peut être considérée comme une opposition de fonction syntaxique, au sens où ces constituants sont définis par leur position dans la phrase. On a donc une répartition du type :

\begin{tabular}{|l|l|l|}
\hline complément / ajout \} intraphrastique & vs & incident \} extraphrastique \\
\hline
\end{tabular}

6 et la différence entre les trois appellations correspond aux propriétés syntaxiques suivantes :

\begin{tabular}{|l|l|}
\hline > complément (de verbe) : & $\begin{array}{l}\text { parfois supprimable } \\
\text { non déplaçable } \\
\text { pronominalisable } \\
\text { clivable } \\
\text { sous la portée de l'interrogation ou de la négation }\end{array}$ \\
\hline
\end{tabular}

\begin{tabular}{|l|l|}
\hline > ajout de SV & $\begin{array}{l}\text { toujours supprimable } \\
\text { difficilement déplaçable (sauf contraste) } \\
\text { non pronominalisable } \\
\text { clivable } \\
\text { sous la portée de l'interrogation ou de la négation }\end{array}$ \\
\hline
\end{tabular}

\begin{tabular}{|l|l|}
\hline > ajout de phrase & $\begin{array}{l}\text { toujours supprimable } \\
\text { facilement déplaçable } \\
\text { non pronominalisable } \\
\text { clivable } \\
\text { sous la portée de l'interrogation ou de la négation }\end{array}$ \\
\hline
\end{tabular}

\begin{tabular}{|l|l|}
\hline > incident & $\begin{array}{l}\text { toujours supprimable } \\
\text { facilement déplaçable } \\
\text { non pronominalisable } \\
\text { non clivable } \\
\text { hors de la portée de l'interrogation ou de la négation }\end{array}$ \\
\hline
\end{tabular}

\begin{tabular}{|c|c|c|c|c|c|}
\hline \multicolumn{3}{|c|}{ Constituants (intraphrastiques) } & \multicolumn{3}{|c|}{ Incident (extraphrastique) } \\
\hline + clivage & $\begin{array}{l}\text { + portée } \\
\text { interr }\end{array}$ & $\begin{array}{l}\text { + portée } \\
\text { nég }\end{array}$ & - clivage & $\begin{array}{l}\text { - portée } \\
\text { interr }\end{array}$ & $\begin{array}{l}\text { - portée } \\
\text { nég }\end{array}$ \\
\hline
\end{tabular}




\begin{tabular}{|l|l|l|}
\hline \multirow{2}{*}{ > complément } & $\begin{array}{l}\text { +/- supprimable } \\
\text { - déplaçable } \\
\text { + pronominalisable }\end{array}$ & \\
\hline > ajout de SV & $\begin{array}{l}\text { + supprimable } \\
+ \text { /- déplaçable } \\
\text { - pronominalisable }\end{array}$ & $\begin{array}{l}\text { + déplaçable } \\
\text { - pronominalisable }\end{array}$ \\
\hline > ajout de P & + supprimable & \\
& + déplaçable & \\
\hline
\end{tabular}

7 Ainsi, dans Selon toute vraisemblance, les étudiants inscriront leur nom dans les délais, l'incident selon toute vraisemblance se reconnaît au fait qu'il n'a aucune des propriétés des constituants (intraphrastiques) : *C'est selon toute vraisemblance que les étudiants inscriront leur nom dans les délais, *Les étudiants inscriront-ils leur nom dans les délais selon toute vraisemblance (ou en toute hypothèse) ? *Les étudiants n'inscriront pas leur nom dans les délais selon toute vraisemblance (mais en toute hypothèse). En revanche, leur nom d'une part, dans les délais d'autre part, se prêtent à ces tests, mais ce qui les distingue, c'est que leur nom n'est pas supprimable, mais il est cliticisable (c'est un complément de verbe), tandis que dans les délais est supprimable, non cliticisable, mais difficilement déplaçable (c'est un ajout de SV). On peut adjoindre à la phrase avec l'Internet: non cliticisable mais très facilement déplaçable, ce syntagme prépositionnel serait analysable comme un ajout de $\mathrm{P}$.

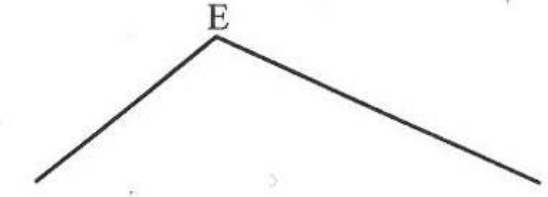

SP

Selon toute vraisemblance

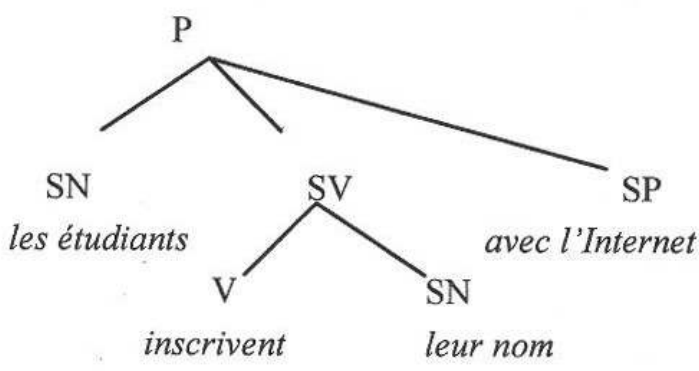

8 On admet que le terme argument concerne un constituant régi: seul le verbe peut donc avoir un complément (les autres constituants venant s'ajouter au syntagme ou à la phrase).

9 Comparons le SP à la radio dans un énoncé comme Il a appris à la radio la démission $d u$ premier ministre, qui peut recevoir trois interprétations possibles :

a. «C'est la radio qui lui apprend la nouvelle, il l'apprend par la radio ». Pour cette première interprétation, le test de la suppression est possible: Il a appris la démission du premier ministre, en revanche le déplacement pose quelque problème:??A la radio, il a appris la 
démission du premier ministre et la cliticisation est impossible, *Il lui a appris la démission du premier ministre ne correspondant pas à l'interprétation (a)

b. «C'est lui qui a informé la radio, il a téléphoné la nouvelle à la radio ». En l'occurrence, la suppression Il a appris la démission du premier ministre ne rend pas compte de l'interprétation (b) mais de celle de (a), et le déplacement pose également des problèmes : ? ? la radio, il a appris la démission du premier ministre - le bloc à la radio n'est pas déplaçable sans effet de contraste, quelque chose comme A la radio, il a appris la démission du premier ministre, mais au journal, il n'a rien dit. Ici, la cliticisation est possible en maintenant la même interprétation : Il lui a appris la démission du premier ministre.

c. « Il se trouvait à la radio quand la nouvelle a été annoncée ». Cette troisième interprétation autorise le test de suppression (Il a appris la démission du premier ministre) et celui du déplacement (A la radio, il a appris la démission du premier ministre) mais exclut la cliticisation.

10 Ainsi en (b) à la radio est un argument; le bloc définit la construction du verbe (on apprend quelque chose à quelqu'un), tandis qu'en (a) et (c) le test du déplacement impossible en (a) et possible en (c) signifie qu'il faut deux analyses différentes pour ce qui n'est pas argument. On dira alors qu'en (c) à la radio est un ajout de phrase et qu'en (a) à la radio est ajout de SV (moins mobile, donc moins autonome par rapport au verbe). C'est ce qui motive la tripartition actuellement observée.

\section{Les limites de ces critères}

11 Cette reconfiguration ne permet cependant pas toujours de trancher clairement entre les différents types de constituants. Par exemple :

12 2.1. L'opposition entre argument et ajout de SV est problématique, puisqu'il y a des SP qui ne sont pas supprimables (donc normalement analysables comme des arguments), mais également non cliticisables (donc normalement analysables comme des ajouts); ainsi dans les phrases [1], [2], [3] :

[1] Marie pleure après une augmentation

[2] Il faut toujours que tu pleures sur ton sort

[3] Le client pleure que le service n'est pas assez rapide

[1.a] *Marie pleure

[2.a)] *I faut toujours que tu pleures

[3.a] * Le client pleure

ni n'est non plus cliticisable :

[1.b] * Marie y pleure/en pleure/la pleure

$[2 . \mathrm{b}]{ }^{*} \mathrm{Tu}$ y pleures

[3.b] * Le client le pleure

15 La non-suppression (critère suffisant pour révéler un "argument » selon O. Bonami 1999) ne va donc pas de pair avec la cliticisation. On peut alors distinguer sur la base de la non-suppressibilité entre complément d'objet (cliticisable) :

[4] Je consens à cette sortie

[4.a] J'y consens

[5] Il embrasse les femmes

[5.a] Il les embrasse 


\section{[6] Il embrasse les femmes du regard}

[6.a] *Il en embrasse les femmes

La notion de "complément oblique » permet de résoudre le problème posé par les compléments (non supprimables, donc forcément régis) qui n'ont pas la propriété d'être cliticisables (comme les compléments d'objet).

2.2. La distinction entre ajout de SV et ajout de $\mathrm{P}$ peut aussi poser des problèmes dans la mesure où elle est fondée sur le critère du déplacement plus ou moins aisé (l'intuition sur la possibilité de déplacement peut varier, selon les individus et selon les contextes). Par exemple, je ne dirais pas :

[6b] *Du regard, il embrasse les femmes

[6.c] ?Du regard, il embrassa le vaste champ de ruines qui s'offrait à ses yeux

Les locatifs posent également des problèmes, puisque les arguments (7.a) au même titre que les ajouts (8.a) sont pronominalisables :

[7] Je vais au marché

[7.a] J'y vais

[8] Paul mange une pomme dans le jardin

[8.a] Paul y mange une pomme

On voit que l'appellation traditionnelle «circonstanciel de lieu» recouvre des comportements syntaxiques différents : si, étant donné la reconfiguration proposée, on peut maintenant considérer sur la ville dans L'ennemi marche sur la ville comme un complément oblique (non supprimable et non cliticisable), comment continuer à justifier que le clitique, censé caractériser le complément d'objet, soit susceptible de concerner des ajouts? Qui plus est, le $y$ de J'y consens n'est pas le même que le $y$ de J'y vais (les anciennes grammaires distinguaient d'ailleurs entre "pronom personnel » (pour le premier) et «pronom adverbial» (pour le second) : le premier n'alterne pas, comme le second, avec là ou bien où (Je vais là / *Je consens là, Où vas-tu ? / Où consenstu ?) - de même, d'ailleurs, qu'entre deux en, celui de Elle rêve de vacances / Elle en rêve / Elle rêve de ça / De quoi rêve-t-elle? et celui de Elle arrive de Dijon / Elle en arrive / Elle arrive de là / D'où arrive-t-elle? Une double difficulté non résolue subsiste donc :

- l'interprétation syntaxique à donner à la propriété de cliticisation (on ne peut pas maintenir que le clitique révèle un complément, puisque, non seulement les constituants non supprimables ne sont pas forcément cliticisables, mais que, de surcroit, des ajouts s'avèrent l'être) ;

- l'analyse des locatifs, seuls à être sujets à cliticisation, alors même qu'ils sont ajouts (de SV aussi bien que de P) - analyse justifiée par le fait qu'ils ne sont pas nécessairement compris dans la reprise en le faire; ainsi dans le salon dans Ils prennent le thé dans le salon autorise l'enchaînement Ils prennent le thé dans le salon et nous le faisons dans la cuisine, bien que la cliticisation Ils y prennent le thé soit autorisée.

2.3. La distinction entre ajout de $\mathrm{P}$ et incident est aussi susceptible de poser des problèmes. Ainsi, à mon avis est habituellement considéré comme un incident (ou «adverbe d'énonciation » dans d'autres terminologies) et ne se soumettrait donc pas à l'extraction en c'est que, ni ne serait sous la portée de l'interrogation ou de la négation. Toutefois, on peut se demander s'il est totalement impossible de dire : 
[9] C'est à mon avis que Paul est malade (et pas selon Marie)

[10] C'est de mon point de vue que Paul est malade

[11] Paul n'est pas malade à mon avis, mais de l'avis unanime de tous !

[12] Est-ce (seulement) à ton avis que Paul est malade - ou aussi pour sa femme et ses amis?

Parmi les « circonstants initiaux portant sur l'énonciation » (Le Goffic, op. cit. : 461), à mon avis n'est pas le seul à paraître pouvoir admettre des tests qui normalement caractérisent les constituants intraphrastiques: selon moi, d'après des sources bien informées et d'autres semblent aussi s'y prêter. Une étude précise de ces syntagmes prépositionnels reste donc à mener, leur analyse n'étant pas aussi aboutie que celle des adverbes - qu'on leur a peut-être un peu trop vite plaquée.

\section{Illustration sur le GP [moyen]}

L'identification du GP [moyen] du type à la main, à la machine, à l'eau tiède, au vinaigre, au balai illustré par les exemples suivants :

[13] Marie lave le linge à la main

[14] Marie lave le linge à la machine

[15] Léa a lavé son pull à l'eau tiède

[16] Marie rince ses cheveux au vinaigre

[17] Pierre brosse le mur au balai

soit comme ajout (de P ou de SV) soit comme argument pose également des difficultés dans la mesure où l'ensemble des critères ne convergent pas et où il revient au linguiste de trancher et de privilégier certains critères plutôt que d'autres, sans qu'il lui soit toujours possible de justifier ses choix.

L'analyse syntaxique du SP [moyen] en à est en apparence relativement simple : dans Léa a lavé son pull à l'eau tiède, à l'eau tiède est supprimable (15.a) et non cliticisable (15.b) :

[15.a] Léa a lavé son pull

[15.b)] *Léa y a lavé son pull

donc c'est un ajout. Mais si l'on tient compte des autres critères, la conclusion est plus hésitante ; ainsi, le complément doit être compris dans la reprise en le faire ou être pseudo-clivé avec le verbe (Bonami, op.cit.). Dans ce cas, on devrait alors avoir clairement :

[15.c] ?Ce qu'elle a fait à l'eau tiède, c'est de laver son pull or la formulation apparaît moins acceptable ou naturelle que :

[15.d] Ce qu'elle a fait, c'est de laver son pull à l'eau tiède

29 Cependant, on est en droit de relativiser aussi ce critère, du fait qu'il ne s'applique pas à tous les énoncés (pour des raisons indépendantes de l'analyse en argument $v s$ ajout) :

[18] L'examen a duré toute la journée

[18.a] ? ? Ce que l'examen a fait, c'est de durer toute la journée

[18.b) *Ce que l'examen a fait toute la journée, c'est de durer

30 Restent les tests portant sur le déplacement, qui ne permettent pas de lever l'hésitation, du fait que si l'on peut admettre :

[15.e] ?A l'eau tiède, Léa a lavé son pull 


\section{[15.g] ? ?Léa à l'eau tiède avait lavé son pull}

Or, si l'ajout de SV se déplace difficilement en tête de phrase, il est normalement mobile à l'intérieur du constituant dont il relève (donc [15.f] devrait être possible) ou en tête ou à la fin de ce dernier (donc [15.g] devrait être aussi acceptable que [15], ce qui n'est pas le cas).

En résumé, il est clair que à l'eau tiède n'est pas cliticisable : ce peut donc être un complément oblique ou un ajout; s'il est supprimable, c'est un ajout, mais est-il supprimable? On n'a pas de raison syntaxique de répondre par oui ou par non, mais seulement une intuition sémantique ; or, comme on l'a aperçu en introduction, celle-ci est sujette à caution : doit-on considérer que laver à l'eau tiède équivaut à laver ou que au contraire, il s'agit d'un type de lavage (qui s'opposerait à laver au vinaigre, laver à l'essence) - en se référant aux hypothèses de P. Cadiot (1991) ? Il faut alors passer à d'autres critères : la relative inacceptabilité de [15.c)] comparé à [15.d] fait pencher la balance en faveur du complément oblique, mais en même temps, la possibilité de laisser le SP en dehors de la reprise en le faire le ferait pencher en faveur de l'ajout : Léa a lavé son pull à l'eau tiède et Paul l'a fait à l'eau froide.

\section{Qu'est-ce qu'un argument / un constituant régi ?}

Si l'on part de la définition de la sous-catégorisation que donne A. Delaveau (2001: 55) :

On appelle compléments sous-catégorisés les compléments dont la présence et le type sont définis dans l'identité lexicale du terme qui les régit?

on est renvoyé à la question de savoir comment on repère l'identité lexicale du terme recteur. Selon cet auteur, il s'agit des différentes constructions associées aux différentes significations. Si l'on consulte le Grand Larousse de la Langue Française ou le Trésor de la langue française, on a effectivement différentes acceptions qui font l'objet de rubriques $(1,2,3 \ldots)$ et de gloses différentes, par exemple pour le verbe abattre :

37 1. abattre un mur

2. abattre un animal

3. abattre le despotisme

4. abattre du travail, etc.

Néanmoins, comme on le voit, les ouvrages lexicographiques n'arborent pas chaque fois des «constructions » différentes, puisque le verbe est, dans tous les cas, défini comme associé à un $\mathrm{SN}$; à strictement parler, la sous-catégorisation est la même: ce qui change, c'est le choix du nom effectif, la distribution, donc la sélection (mur ou animal: le sens diffère pour le verbe, «l'action " n'est pas la même, la commutation ne s'opère pas avec les mêmes synonymes, etc.). Si l'on suit la citation d'A. Delaveau, on aurait une identité lexicale ? Le même verbe, puisque la même construction? On s'aperçoit en tous cas que l'on n'a pas biunivoquement un sens qui correspond à une construction : sur quoi alors fonde-t-on « l'identité lexicale »? 
39 Mais les dictionnaires ne signalent pas, par exemple, que, selon le type de nom, donc selon le sens que prend le verbe, on a ou non la possibilité d'un SP à sens « moyen »; par exemple, on peut :

1 '. abattre un mur à la masse

2'. abattre un arbre à la hache

mais on ne trouve pas parallèlement :

42 3'. abattre le despotisme à la... ( ?)

4'. abattre du chemin / des kilomètres à la... ( ?)

5'. abattre son jeu / ses cartes à la... (?)

43 De même abattre ou massacrer sont décrits comme abandonner ou aimer, si l'on s'en tient au SN :

[19.a] Elle a massacré son amant

[20.a] Elle a abandonné son amant

44 Or, seul le premier permet l'apparition d'un moyen :

[19.b] Elle a massacré son amant à la tronçonneuse

[20.b] *Elle a abandonné son amant à la... ( ?)

Si cette observation est exacte en a) et en b), abattre est caractérisé non pas seulement par la construction $\mathrm{V}+\mathrm{SN}$ mais en fait aussi par la construction $\mathrm{V}+\mathrm{SN}+\mathrm{SP}$ où $\mathrm{SP}$ dénote un certain type de moyen. Et de même, selon ce critère, massacrer et abandonner n'impliquent pas les mêmes sous-catégorisations : intuitivement d'ailleurs, massacrer suppose un moyen mais non abandonner. Un autre argument en ce sens est que abattre un oiseau et abattre un cheval ont en fait deux interprétations, la première «tuer » et la seconde où abattre peut également signifier « coucher l'animal pour le soigner » (TLF) : si le vétérinaire abat un cheval, c'est pour l'opérer, par exemple (et dans ce cas, le verbe n'est pas assorti du SP [moyen]: la possibilité d'un tel SP distingue donc bien deux emplois de abattre).

Par conséquent, si le complément est défini comme un constituant régi (en l'occurrence par le verbe), et si la rection est définie comme la détermination de ce qui, dans un emploi donné de la tête, entre dans sa construction syntaxique et sémantique (laquelle constitue son identité lexicale), alors le SP [moyen] en à (de type à la hache) est bien un complément de abattre (dans Max abat l'arbre à la hache) : c'est syntaxiquement ce qui distingue abattre un arbre de abattre du chemin ou abattre le despotisme, l'alternance un arbre / du chemin / le despotisme relevant de la sélection et non de la sous-catégorisation. De même pour un énoncé comme Laver à la main (exemple [13] vs Laver à la machine (exemple [14]) où la spécification (à la main vs à la machine) paraît délimiter deux actions bien différentes : en effet, si je lave mon linge à la machine, cela n'implique pas que je le lave (moi-même), ce qui n'est pas le cas si je le lave à la main : à la main serait supprimable mais non à la machine! Mais alors on ne peut plus définir globalement « un » constituant de type «SP [moyen en à] » puisque, selon les verbes et les emplois de verbes, il serait tantôt complément, car non supprimable (à la machine) et tantôt ajout, car supprimable (à la main). L'analyse serait donc à mener au cas par cas avant toute généralisation - ce que recommandait déjà M. Gross (1975 \& 1976) à l'encontre des procédures en vigueur en grammaire générative.

Il y aurait donc deux analyses syntaxiques possibles du SP de moyen en à : ou bien il participe de l'identité lexicale du verbe, en ceci que ce dernier a des emplois qui 
permettent la présence d'un tel SP et d'autres qui l'excluent (abattre un cheval au sens «tuer » vs abattre un cheval au sens " coucher l'animal ») - et dans ce cas il s'agit d'un complément (oblique, puisque la cliticisation n'est pas permise); ou bien la présence opposée à l'absence du SP ne sépare pas des emplois nettement distincts (laver son pull aussi bien que laver son pull à l'eau tiède/au vinaigre / à la main / à la machine, c'est toujours « débarrasser le pull de la saleté ») - auquel cas il s'agit d'un ajout.

\section{Conclusion}

L'analyse grammaticale s'est peu à peu spécifiée, dans les cinquante dernières années, d'une part en donnant la priorité aux critères syntaxiques, d'autre part en découvrant de nouvelles propriétés susceptibles de caractériser les fonctions. En particulier dans le domaine intraphrastique, qui concerne plus spécialement le SV, a-t-on abouti à la configuration suivante :

\begin{tabular}{|c|c|c|c|}
\hline & \multicolumn{2}{|l|}{ Complément (régi) } & \multirow[t]{2}{*}{ Ajout } \\
\hline & Complément d'objet & Complément oblique & \\
\hline Suppression & $+/-$ & - & + \\
\hline Déplacement & - & - & +/- \\
\hline Cliticisation & + & - & - \\
\hline Compris dans le faire & + & + & - \\
\hline
\end{tabular}

Théoriquement, le tableau permet de distinguer les trois constituants ; en pratique, on se heurte à diverses difficultés : le test de la suppression suppose le recours à l'intuition sémantique, donc n'apparaît pas toujours fiable ; la cliticisation n'est en fait pas propre au complément, pouvant concerner l'ajout locatif; les acceptabilités varient quant au déplacement ; la reprise en le faire est très dépendante de l'identité du verbe lui-même.

\section{BIBLIOGRAPHIE}

BLANCHE-BENVENISTE, C. ET COLL. (1984), Pronom et syntaxe. L'approche pronominale et son application au français, Paris, Selaf et Aelia.

BONAMI, O. (1999), Les constructions du verbe : le cas des groupes prépositionnels argumentaux, Thèse de doctorat, Université Paris 7.

BOONS, J.P., GUILLET, A. \& LECLERE, C. (1976), La structure des phrases simples en français : constructions intransitives, Genève, Droz. 
CADIOT, P. (1991), « A la hache ou avec la hache ? Représentation mentale, expérience située et donation du référent », Langue française, 91, pp. 7-23.

CADIOT, P. (1993), « De et deux de ses concurrents : avec et à », Langages, 110, pp. 68-106.

CADIOT, P. (1997), Les prépositions abstraites en français, Paris, Armand Colin.

CERVONI, J. (1991), La préposition. Etude sémantique et pragmatique, Paris, Duculot.

CHEVALIER, J.-C. (1968), Histoire de la syntaxe. Naissance de la notion de complément dans la grammaire française (1530-1750), Genève, Droz.

DELAVEAU, A. (1992), « Les compléments circonstanciels et l'analyse syntaxique », Le gré des langues, 3, pp. 188-201.

DELAVEAU, A. (2001), Syntaxe : la phrase et la subordination, Paris, Armand Colin.

DUBOIS-CHARLIER, F. (2001), " Complément de verbe, de proposition, de phrase, d'énoncé », Travaux, 17, Cercle de Linguistique d'Aix-en-Provence, pp. 33-49.

GROSS, M. (1975), Méthodes en syntaxe, Paris, Hermann.

GROSS, M. (1976), « Présentation », dans J.-P. Boons, A. Guillet \& C. Leclère (éds), pp. 7-28.

GUIMIER, C. [dir] (1993), 1001 circonstants, Caen, Publications de l'Université de Caen.

GUIMIER, C. (1996), Les adverbes du français, Paris, Ophrys.

HAMON, S. \& LAVIEU, B. (2002), « Complément de verbe et modifieur : quelles propriétés ? ", Communication à la journée d'étude Unités lexicales : propriétés morphologiques et propriétés syntaxiques, Université Paris X-Nanterre, 14 juin 2002.

HAMON, S. (2005), La phrase double causale ; propriétés syntaxiques et interprétations sémantiques, Thèse de doctorat, Université Paris X-Nanterre.

HUDDLESTON, R. \& PULLUM, G. K. (2002), The Cambridge Grammar of the English Language, Chap. 4 "The clause complements", Cambridge University Press, pp. 215-321.

LAVIEU, B. (2005), Le $\mathrm{SP}_{\text {moyen }}$ introduit par la préposition 'à' : analyse syntaxique, sémantique et lexicale, Thèse de doctorat, Université Paris X-Nanterre.

LE GOFFIC, P. (1993), Grammaire de la phrase française, Paris, Hachette Université, coll. “Langue française".

LEEMAN, D. (1985/1986), « A propos du complément circonstanciel », Travaux de linguistique, 12/13, pp. 177-193.

LEEMAN, D. (1997), « Définir une préposition : hypothèses et perplexités », Revue de Sémantique et Pragmatique, 2, pp. 183-199.

LEEMAN, D. (1998), Les circonstants en question(s), Paris, Kimé.

LEEMAN, D.. (2002), La phrase complexe. Les subordinations, Bruxelles, De Boeck.

LEEMAN, D. (2005), Cours de Syntaxe : Grammaire, Université de Paris X, Comète.

MELIS, L. (2003), La préposition en français, Paris, Ophrys (Collection « L'essentiel français »). TOURATIER, C. (2001), « La notion de circonstant », Travaux, 17, Cercle de Linguistique d'Aix-enProvence, pp. 17-31.

VAGUER, C. (2004), Les constructions verbales 'V dans GN'. Approches syntaxique, lexicale et sémantique, Thèse de l'Université Paris X-Nanterre. 
VANDELOISE, C. (1986), L'espace en français : sémantique des prépositions spatiales, Paris, Le Seuil.

VANDELOISE, C. (1987), « La préposition à et le principe d'anticipation », Langue française, 77, pp. 77-111.

VANDELOISE, C. (1988), « Les usages spatiaux statiques de la préposition à », Cahiers de lexicologie, 53, pp. 119-148.

VANDELOISE, C. (1993), « La préposition à pâlit-elle derrière toucher ? », Langages, 110, pp. 107-127.

VARGAS, C. (2001), « Compléments, circonstants et circonstanciels dans quelques grammaires universitaires du français », Travaux, 17, Cercle de Linguistique d'Aix-en-Provence, pp. 51-72.

WAGNER, R. L. \& PINCHON, E. (1962), Grammaire du français classique et moderne, Paris, Hachette supérieur, coll. "H.U. Langue française".

\section{NOTES}

1. Je remercie Dany Amiot, Danielle Leeman, Pierre Péroz et Céline Vaguer pour leurs remarques sur le texte initial, présenté lors du colloque La préposition comme valeur, Florence, 5-7 avril 2006.

2. Exemple cité par Boons, Guillet, Leclère (1976).

3. Exemple cité par Dubois-Charlier (2001).

\section{RÉSUMÉS}

Belinda Lavieu retrace les différentes étapes de l'approche de la fonction de complément, depuis la répartition traditionnelle entre "objet» et "circonstanciel», ensuite la distinction structuraliste entre " essentiel » et «non essentiel », jusqu'à nos jours où le « complément » est distingué de l' "ajout » et de l' « incident », le complément recouvrant le « complément d'objet » et le « complément oblique ». L'inventaire des propriétés disponibles et leur application montre néanmoins leurs limites : la recherche n'est pas encore parvenue à une classification fiable.

\section{AUTEUR}

\section{BELINDA LAVIEU}

Université de Paris X - Nanterre 